\title{
Nurses' View towards the Use of Robotic during Pandemic COVID-19 in Indonesia: A Qualitative Study
}

\author{
Taryudi Taryudi $^{1 *}$ (D), Linlin Lindayani ${ }^{2}$ (D) Heni Purnama ${ }^{2}$ (D) Astri Mutiar ${ }^{2}$ (D) \\ ${ }^{1}$ Department of Electrical Engineering, Faculty of Engineering, Univeristas Negeri Jakarta, Jakarta, Indonesia; ${ }^{2}$ Department of \\ Nursing, Sekolah Tinggi IImu Keperawatan PPNI Jawa Barat, Bandung, Indonesia
}

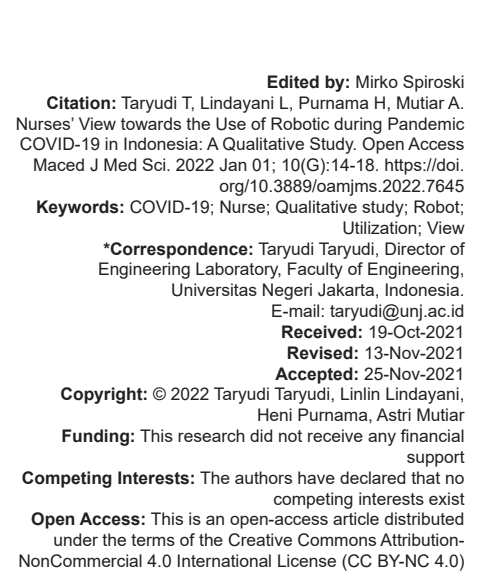

\section{Introduction}

Robots are rapidly being used in healthcare, alleviating difficulties for patients, hospitals, and the industry as a whole [1]. Rapid advances in artificial intelligence and robotics have resulted in an explosion of intelligent technologies integrated into our domestic and industrial settings. However, the health care system is identical to human-centered, and many healthcare professions may not be ready to work with robots [2]. However, because the devices have the potential to improve outcomes, it is critical to address how healthcare practitioners learn about and engage with robots, share workspaces, and collaborate to obtain healthcare outcomes [3].

Several collaborative robots were created during COVID-19, including one in Florida that can stop an intravenous pump device, change the ventilator knob, and stop the ICU monitor when an occlusion is detected [4]. In Indonesia, the robot RAISA was developed by the November 10 Institute of Technology and Airlangga University Hospital to help provide some care for patients with COVID-19 to minimize contact between medical personnel and COVID-19 patients. Previous study compared the opinions of Finnish and Japanese eldercare staff and reported that the Japanese workers thought robots were is beneficial) [5]. The study discovered substantial differences in care staff's perceptions in two countries about the usefulness of possible robot tasks. Understanding nurses' views toward robotics can help integrate robotic technologies into future patient care [6]. In addition, cultural views toward robots vary and must be considered while developing robotic products for different countries and civilizations [6]. This study aimed to explore how nurses view using robotics during the COVID-19 pandemic.

\section{Methods}

\section{Study design}

This study used a qualitative descriptive technique to understand better the significance of human experience [7]. Qualitative descriptive research 
has been emphasized as critical and beneficial for elucidating who, what, and where of events and experiences and eliciting viewpoints from respondents about a poorly defined issue [7]. Between April and May 2021, this study was undertaken at two COVID-19 referral hospitals in West Java, Indonesia. When compared to other Java regions, the number of COVID-19 cases in West Java, Indonesia, rose significantly over the study period.

\section{Sample}

All participants were registered nurses who provide direct care to the patients with COVID-19 recruited from two hospitals in Indonesia. Because these hospitals have not deployed robots yet, individuals may express their views openly. Purposive sampling was used to select respondents with criteria of those who had worked for at least 1 year and were willing to participate.

Sample size was determined based on data saturation, defined as the point at which no new themes emerged [8].

\section{Interview guideline}

The interview guideline was developed based on a related literature review and expert discussion. The question included: (1) What is your perspective on the usage of robots during the COVID-19 pandemic? (2) Can you envision and describe how your jobs might evolve if your unit implements robots soon? (3) What might change in care be expected in the clinical setting if robots are used to combat pandemic COVID-19? (4) What are the consequences of using robotic care?

\section{Data collection}

Human Research Ethics Committees of the affiliated university gave their approval to conduct the study (III/0132/KEPK/STIKEP/PPNI/JABAR/V/2021). The nurse managers helped to inform potential participants. Potential participants were contacted through WhatsApp and sent an invite and an information page outlining the study objectives. After obtaining consent from potential participants, researchers and participants scheduled a face-to-face interview. Each interview lasted about 45-60 min. The interviews were done using a semi-structured interview guide with openended questions. Data were collected until a saturation point of 20 participants was reached.

\section{Data analysis}

The analysis used in this study was qualitative content analysis. Lincoln and Guba (1985) emphasized the importance of rigor in enhancing the reliability of findings [9]. All participant interviews were taped and verbatim transcribed to reduce bias. The analysis was led by an experienced qualitative research method (LL), in which two additional investigators assisted in data processing and interpretation. The first step was to read the verbatim text for simple phrases as outlines using open coding and then gather headings onto coding sheets and generated categories [10]. Each category was designated using content-specific terms; subcategories containing related events and situations were combined to form categories; subthemes were further divided into the theme [11].

\section{Results}

A total of 20 female nurses with an average age of $32.8 \pm 4.0$ years participated in this study. Three were magister in nursing, 10 were a bachelor in nursing, and 7 were diploma three in nursing. Four were head nurses, and 16 were staff nurses. All participants were currently working in the COVID-19 unit. The qualitative findings revealed three themes with nine sub-themes: the use of robotic in nursing care, the burden of using robotic in nursing care, and attitude toward robotic in nursing care (Table 1).

Table 1: Theme and sub-themes emerged in the study

\begin{tabular}{ll}
\hline Theme & Sub-theme \\
\hline The useful of robotic in nursing care & $\begin{array}{l}\text { Reducing the risk of COVID-19 transmission } \\
\text { Monitoring patients remotely } \\
\text { Helping in providing care } \\
\text { Digital literacy in nursing care }\end{array}$ \\
$\begin{array}{ll}\text { The burden of using robotic in nursing } \\
\text { care }\end{array}$ & $\begin{array}{l}\text { Cultural difference in providing care } \\
\text { Changing care practice habits safety concern } \\
\text { Attitude toward robotic in nursing care }\end{array}$ \\
\hline
\end{tabular}

\section{Theme 1: the use of robotic in nursing care}

Nurses are at the battle's forefront during pandemics and a professional group with high vulnerability. Nurse views the use of robots in caring for patients with COVID-19 was to reduce the transmission of COVID-19.

“... in my opinion it could help us reduce risk of getting COVID-19, I hope we have such kind of robotic in our hospitals." (N12, 39 years old, nurse supervisor)

"I think we work together with robot to help us and it will at least lower our risk to get COVID-19". (N5, 32 years old, nurse practitioner)

Monitoring patients remotely view as one of the useful of nurse robotics to be implemented in the hospital. Considering the patient-nurse ratio during the pandemic, COVID-19 was high, but all the patients with COVID-19 need to be monitored intensively. The presence of robots will greatly help nurses in this regard.

"We need to monitor patients remotely because they were in isolation room. If we. have nurse robotic, 
we can monitor and talk to patients remotely. That's will be great" (N15, 29 years old, nurse supervisor).

The nurse also thought that robots could help provide care by helping their minimal contact with patients in providing medication, quick response to the patient needs, or vital sign assessment. Thus, it may reduce their workload and make their work easier.

"Nurse robot can help us to provide drug for patient isolation room, or check vital sign may be...". (N10, 34 years old, nurse practitioner)

"Patients COVID-19, many of them are being fear and anxiety so they are very often to call us. I think we work together with robot to help us, especially during this pandemic providing quick response if patients ask for help." (N3, 27 years old, nurse practitioner)

"I hope by having robot can reduce our workload at least for some task" (N1, 28 years old, nurse practitioner)

"If we have robot that can help nurse to move patients like in Japan as I saw in the internet, I will not suffer from back pain anymore, I think! (laugh)." (N8, 40 years old, nurse practitioner)

\section{Theme 2: Burden of using robotic in}

\section{nursing care}

The use of robotic in providing care to a patient with COVID-19 gained burse to nurses. Nurse needs to improve their digital literacy and competency in using technology. This is needed to be able to operate the robot and maximize its function in helping nursing services.

"It is means that I have to upgrade our knowledge in using technology, keep updating current information that might be little hard to do." (N4, 35 years old, nurse practitioner)

Nursing care is identical to human care. For others, the presence of robots may be viewed as harmful because the information shown in films about robots is identical to frightening things that can endanger human life. As a result, patients and their families may refuse to receive assistance from robots.

"In movie, robot is scary, they will destroy human life, this condition will affect to the patent and family acceptability in receiving care from robot." (N15, 29 years old, nurse supervisor)

The usage of robots in nursing will also result in a shift in nurses' routines working in hospitals. Nurses must be socialized and internalized to accept and work with robots. Nurses must adapt to working with machines and dividing up the task.

"It's one challenge that we could try hard to adapt, change our routine work, and working together with robot." (N4, 35 years old, nurse practitioner)

"As long as we have clear standard operational procedure in utilizing robot that will be Ok even, we need some time to get familiar." (N9, 29 years old, nurse supervisor)

"Hmmm.... it's challenging actually even its good, but we have to get training in advance to handle robot in the care unit." (N7, 32 years old, nurse practitioner)

Nurse highlights the importance of safe and accurate robotic systems.

"They should be safe for sure without error, and if it's possible the robot better have long battery." (N11, 30 years old, nurse practitioner)

"Their (robot) precises work is believable but still possible to have error in the machine." (N4, 29 years old, nurse practitioner)

\section{Theme 3: Attitude toward robotic in nursing care}

Participants reported a negative toward robots' usefulness and benefits in providing care during the COVID-19 pandemic. The negative attitude was related to believing that the robot could replace the nurse work in the future. Using robots could reduce nurse skills, afraid that patients will choose robots over humans.

"If we radically develop robot in healthcare without considering human labor, probably in the future our work could be replace by robot." (N4, 38 years old, nurse practitioner)

"How if patients more likely to be cared by robot? hmmm ... possible because robot may perform the task without error." (N11, 34 years old, nurse practitioner)

\section{Discussion}

The findings indicate that the use of robotic in nursing care; nurses believed robotics offered numerous benefits. They thought robotics could help reduce COVID-19 transmission, monitor patients remotely, and help provide care. These findings confirm the research related helpfulness and precision to favorable attitudes of robot [12], [13]. Robotics helps provide nursing care to COVID-19 patients. First, robots can help with repetitive activities, precise medicine delivery, remote patient monitoring, and reducing nurses' workload. Several studies have shown the use of robotic during the pandemic COVID-19, included: (1) sanitation and sterilization of hospital facilities, (2) drug distribution, (3) telemedicine and remote help, and (4) new case detection [14], [15], [16]. Furthermore, robots offer great social, emotional, and utilitarian value [17].

Utilizing robotic in nursing care also brings burden into their routine care. They considered the 
burden of adopting robotics, such as constantly updating their technological literacy, and the patient and family rejection due to cultural differences. These findings support a prior study [18] that found technology is not reliable without human observation abilities. Users' knowledge and perception are two significant adoption barriers [19], [20] suggests using culture theory to explain people's social interactions with technology and preferences within its usages. This cultural paradigm will improve people's desire for and confidence in medical robotics and other technologies [21]. Nurses are also looking for robotic solutions that are both safe and accurate. As a result, efforts to deploy robotics for COVID-19 must be focused on improving and gaining higher equipment and increasing safety [22], [23]. In addition, to alleviate hesitation toward robotic platforms, it is critical to incorporate healthcare staff in training programs emphasizing the importance and capabilities of robots during a pandemic.

Participants expressed a negative attitude about robots' utility in managing the COVID-19 outbreak. Negative attitudes were associated with the belief that in the future, robots will be able to take the job of nurses, that utilizing robots will lower nurse skills, and that patients will prefer robots to humans. This finding suggests that nurses need to be educated and aware that robots can help them do their jobs better, but they cannot replace them [24]. While conducting technology-focused continuing education for nurses, nursing administers and educators are encouraged to emphasize the integration of culture with technology to meet culturally competent care needs and patient safety. With an intentional approach, using robotics or advanced technology in nursing care can be fostered. Nurses can more fully understand the impact of culture on technology, and vice versa, to provide culturally competent care.

\section{Limitations}

Our study involved a descriptive design, and all of the participants were female nurses. Thus, the study's findings may not be generalizable. Moreover, some of the participants indicated that their exposure to electronic systems was limited. On suggests that future research should use quantitative methods and recruit people from various hospital services, geographies, and professions.

\section{Conclusion}

This study explored nurses' views on the usage of robotics during the pandemic COVID-19. The qualitative findings revealed three themes, including the use of robotic in nursing care, the burden of using robotic in nursing care, and attitude toward robotic in nursing care (sub-theme: negative response). It implies that strategic plan would have many benefits but also limitations, such as nursing care burden, negative attitude, and cultural awareness. Inevitably, greater usage of robotics and Al will impact nursing job roles and work opportunities; yet, it is feasible that technological advancements will enhance nursing work chances by boosting nurses' abilities and functions. Individual care provided by nurses and their expertise and abilities must continue in some form, whether aided by technology or not. Improving nurses' understanding of robotics is critical.

\section{References}

1. Kujat L, John S, College F. How have robotics impacted healthcare? How has open access to fisher digital publications benefited you? Rev J Undergrad Stud Res. 2010;12:6-8.

2. Smith A, Anderson J. Al, robotics, and the future of jobs. Pew Res Cent. 2014;6:51.

3. Homma K, Matsumoto O. Development of a risk assessment assistance tool for robotic care devices. Stud Health Technol Inform. 2017;242:551-7

PMid:28873852

4. English SW, Barrett KM, Freeman WD, Demaerschalk BM. Telemedicine-enabled ambulances and mobile stroke units for prehospital stroke management. J Telemed Telecare. 2021. https://doi.org/10.1177/1357633X211047744 PMid:34636680

5. Coco K, Kangasniemi M, Rantanen T. Care personnel's attitudes and fears toward care robots in elderly care: A comparison of data from the care personnel in Finland and Japan. J Nurs Scholarsh. 2018;50(6):634-44. https://doi.org/10.1111/ jnu.12435 PMid:30354007

6. Papadopoulos I, Koulouglioti C. The influence of culture on attitudes towards humanoid and animal-like robots: An integrative review. J Nurs Scholarsh. 2018;50(6):653-65. https:// doi.org/10.1111/jnu.12422

PMid:30242796

7. Polit DF, Beck CT, Owen SV. Is the CVI an acceptable indicator of content validity? Appraisal and recommendations. Res Nurs Health. 2007;30(4):459-67. https://doi.org/10.1002/nur.20199 PMid:17654487

8. Lincoln YS, Guba EG, Pilotta JJ. Naturalistic Inquiry. Newbury Park: Cal Sage; 1985.

9. Lincoln YS, Guba EG. Naturalistic Inquiry. Vol. 75. Thousand Oaks, CA: Sage; 1985.

10. Burnard P. Teaching the analysis of textual data: An experiential approach. Nurse Educ Today. 1996;16(4):278-81. https://doi. org/10.1016/s0260-6917(96)80115-8 PMid:8936234

11. Elo S, Kyngäs $H$. The qualitative content analysis process. J Adv Nurs. 2008;62(1):107-15. https://doi. org/10.1111/j.1365-2648.2007.04569.x PMid:18352969

12. Best J, Day L, Ingram L, Musgrave B, Rushing H, Schooley B Comparison of robotic vs. standard surgical procedure 
on postoperative nursing care of women undergoing total abdominal hysterectomy. Medsurg Nurs. 2014;23(6):414-21. PMid:26281645

13. lavazzo $\mathrm{C}$, Gkegkes ID. Enhanced recovery programme in robotic hysterectomy. Br J Nurs. 2015;24(16):S4-8. https://doi. org/10.12968/bjon.2015.24.Sup16.S4 PMid:26355452

14. Aymerich-Franch L. Why it is time to stop ostracizing social robots. Nat Mach Intell. 2020;2(7):364. https://doi.org/10.1038/ s42256-020-0202-5

15. Demaitre E. Coronavirus Response Growing from Robotics Companies. Vol. 3; 2020.

16. Cresswell K, Cunningham-Burley S, Sheikh A. Health care robotics: Qualitative exploration of key challenges and future directions. J Med Internet Res. 2018;20(7):e10410. Available from: http://www.jmir.org/2018/7/e10410. [Last accessed on 2018 Mar 15].

17. Ricks DJ, Colton MB. Trends and considerations in robotassisted autism therapy. In: 2010 IEEE International Conference on Robotics and Automation. 2010. p. 4354-9.

18. Wikström AC, Cederborg AC, Johanson M. The meaning of technology in an intensive care unit-an interview study. Intensive Crit Care Nurs. 2007;23(4):187-95.

PMid:17467992

19. Aarts J, Gorman P. IT in health care: Sociotechnical approaches
"To Err is System". Int J Med Inform. 2007;76 Suppl 1:S1-3. https://doi.org/10.1016/S1386-5056(07)00078-0

PMid: 17466251

20. Coombs C. Will COVID-19 be the tipping point for the intelligent automation of work? A review of the debate and implications for research. Int J Inf Manage. 2020;55:102182. https://doi. org/10.1016/j.ijinfomgt.2020.102182

PMid:32836639

21. White PJ, Marston H, Shore L, Turner R. Learning from COVID-19: Design, Age-friendly Technology, Hacking and Mental Models [Version 1; Peer Review: Awaiting Peer Review]; 2020.

22. Otter JA, Yezli S, Perl TM, Barbut F, French GL. The role of "no-touch" automated room disinfection systems in infection prevention and control. J Hosp Infect. 2013;83(1):1-13. https:// doi.org/10.1016/j.jhin.2012.10.002

PMid:23195691

23. Marra AR, Schweizer ML, Edmond MB. No-touch disinfection methods to decrease multidrug-resistant organism infections: A systematic review and meta-analysis. Infect Control Hosp Epidemiol. 2018;39(1):20-31. https://doi.org/10.1017/ ice.2017.226

PMid:29144223

24. Goh PS, Sandars J. A vision of the use of technology in medica education after the COVID-19 pandemic. MedEdPublish. 2020;9(1):49. http://doi.org/10.15694/mep.2020.000049.1 